Purdue University

Purdue e-Pubs

Experimental investigation of steady buoyantthermocapillaryconvection near an evaporating meniscus

\author{
H. K. Dhavaleswarapu \\ Pramod Chamarthy \\ S V. Garimella \\ Purdue University, sureshg@purdue.edu \\ Jayathy Y. Murthy
}

Follow this and additional works at: http:/ / docs.lib.purdue.edu/coolingpubs

Dhavaleswarapu, H. K.; Chamarthy, Pramod; Garimella, S V.; and Murthy, Jayathy Y., "Experimental investigation of steady buoyantthermocapillaryconvection near an evaporating meniscus" (2007). CTRC Research Publications. Paper 15. http://dx.doi.org/10.10631/.2776164

This document has been made available through Purdue e-Pubs, a service of the Purdue University Libraries. Please contact epubs@purdue.edu for additional information. 


\title{
Experimental investigation of steady buoyant-thermocapillary convection near an evaporating meniscus
}

\author{
Hemanth K. Dhavaleswarapu, Pramod Chamarthy, Suresh V. Garimella, ${ }^{\text {a) }}$ \\ and Jayathi Y. Murthy \\ Cooling Technologies Research Center, School of Mechanical Engineering, Purdue University, \\ West Lafayette, Indiana 47907, USA
}

(Received 12 March 2007; accepted 5 June 2007; published online 17 August 2007)

\begin{abstract}
Micro-particle image velocimetry measurements of the three-dimensional (3D) convection patterns generated near an evaporating meniscus in horizontally oriented capillary tubes are presented. Analysis of the vapor diffusion away from the meniscus reveals a zone of intense heat flux near the solid-liquid-vapor junction that creates a temperature gradient along the meniscus. This results in a surface tension gradient which, coupled with buoyancy effects, causes buoyant-thermocapillary convection in the liquid film. The relative influence of buoyancy and thermocapillarity on the flow was investigated for tube diameters ranging from 75 to $1575 \mu \mathrm{m}$. A transition from a pure two-dimensional thermocapillary flow to a 3D buoyant-thermocapillary flow is observed with an increase in tube diameter. For the $75 \mu \mathrm{m}$ tube, a symmetrical toroidal vortex is observed near the meniscus. For larger tubes, buoyancy effects become apparent as they dominate the flow field. The high mass fluxes in smaller-diameter tubes drive stronger vortices. Particle streaks and micro-particle image velocimetry images obtained in multiple horizontal and vertical planes provide an understanding of this three-dimensional flow behavior. A scaling analysis shows the importance of thermocapillary convection in evaporating menisci. (c) 2007 American Institute of Physics. [DOI: $10.1063 / 1.2752477]$
\end{abstract}

\section{INTRODUCTION}

The interfacial surface tension between two immiscible fluids depends on the imposed temperature, electric fields, and the presence of foreign particles. Under the influence of gradients in temperature, electric field, or particle concentration, surface tension stresses are induced that can give rise to interfacial flows. Thomson ${ }^{1}$ explained the occurrence of tears in strong wine as early as 1855 . The phenomenon arises from the depletion of alcohol at the rim of the glass, resulting in greater surface tension at the rim than at the center, which drives the wine to the rim that falls back as drops along the film. Marangoni ${ }^{2}$ concluded based on Thomson's result that convection could be driven by surface tension gradients due to a liquid's composition or temperature gradients. Hershey ${ }^{3}$ proposed that the higher evaporation or condensation along the border of a liquid leads to local temperature gradients which induce surface tension inequalities and generate liquid motion. Benard's experimental observation of cellular flow ${ }^{4}$ associated with interface deformation in a horizontal liquid layer heated from below led Rayleigh ${ }^{5}$ and Block $^{6}$ to propose different mechanisms for the convection; the former attributed the effect to buoyancy-driven convection and the latter to surface tension-driven convection. The convective motion arising due to the surface tension variation with temperature was termed the thermocapillary effect. ${ }^{7}$ This effect is characterized by the Marangoni number (Ma). ${ }^{8}$ The temperature variation along an interface can be a result of non-

\footnotetext{
${ }^{a)}$ Author to whom correspondence should be addressed. Telephone: 765494-5621. Electronic mail: sureshg@purdue.edu
}

uniformities in evaporation, or heating. Most prior work in this area has been based on nonuniform heating in various geometries such as a layer of fluid, ${ }^{9}$ climbing films, ${ }^{10}$ and liquid bridges. ${ }^{11}$ The present work considers steady convection caused due to nonuniform evaporation of a homogenous liquid along a curved free surface that has wide applications.

Evaporation from a meniscus serves as the basic mechanism in many two-phase cooling devices such as heat pipes, thermosiphons, vapor chambers, two-phase cold plates, and capillary pumped loops. Thin-film evaporation, ${ }^{12}$ which takes place near a solid-liquid-vapor junction, has long been believed to be the dominant mode of heat transfer in such systems. The efficacy of heat transfer in thin films is attributed to a high liquid pressure (termed as disjoining pressure by Derjaguin ${ }^{13}$ ) gradient, which results in liquid being pulled into the thin-film region, as well as the very low thermal resistance resulting from the small film thickness. Wayner and co-workers ${ }^{14,15}$ carried out extensive theoretical and experimental studies in this field and delineated several important factors influencing thin-film evaporation. Ma and Peterson ${ }^{16,17}$ developed experimentally verified models for evaporation from $\mathrm{V}$-grooves to predict its maximum heat transport limit. The intensive heat transfer in the thin-film region is also important to the mechanism of bubble growth during boiling, ${ }^{18}$ film dryout, ${ }^{19}$ and spray cooling. ${ }^{20}$

Evaporation of a liquid even into air-vapor mixtures is of industrial significance in several problems involving falling films, ${ }^{10}$ drying applications, ${ }^{21}$ and cryogenic liquids in spacecraft tankage. ${ }^{22}$ The evaporated vapor is transferred primarily by diffusion and also by convection into the air-vapor regime. In drying from porous media, because of the film cur- 
vature in pores, more intensive evaporation has been shown to occur near the film tips than at other locations along the meniscus. ${ }^{21}$ Differential evaporation along an interface is thus widely prevalent in various evaporation systems.

Thermocapillary convection has been studied qualitatively using tracing particle techniques ${ }^{23}$ and by laser Doppler velocimetry. ${ }^{24}$ Wozniak et al. ${ }^{25-27}$ were the first to apply particle image velocimetry (PIV) and laser-speckle velocimetry as tools to quantify the entire flow field due to thermocapillary convection and buoyancy effects around a bubble. They observed that under microgravity conditions, the surface tension effects dominate and penetrate deep into the bulk, whereas under terrestrial conditions, a compact vortex was seen due to interaction between thermocapillarity and buoyancy. Pline et al. ${ }^{28}$ used two-dimensional (2D) particle displacement tracking to study transient and steady fluid flows in silicone oil subject to inhomogeneous heating in an open circular container. Kamotani et al. ${ }^{29}$ were the first to perform flow visualizations using particle tracking velocimetry in flat and curved free liquid surfaces. They concluded that it was more difficult for the surface flow to penetrate corner regions in curved surfaces than in the case of flat surfaces because of the narrow passage in the cusped region. They also observed a small recirculation cell but were not able to resolve details in this region. Much of the early experimental work in this field was limited in being able to accurately resolve the flow field close to the surface.

Boiling heat transfer is also strongly affected by the dynamic flow field surrounding bubbles. Savino and Fico ${ }^{30}$ conducted a combined experimental and numerical analysis on the behavior of bubbles on a horizontal heated surface. They concluded that the heat transfer from the wall is enhanced by a factor of 4.5-5 because of the cold-return flow caused by thermocapillary convection. They also demonstrated the change in convection-enhanced heat transfer under zero gravity and terrestrial conditions, highlighting the importance of buoyancy effects. Under low Marangoni numbers, the temperature and velocity fields were found to be steady, while at very high Marangoni numbers, oscillations were observed. Ward and Duan ${ }^{31}$ examined thermocapillary convection due to steady evaporation of water in a conical vessel. They concluded that $50 \%$ of the energy transport occurs due to mixing effects. Ruiz and Black ${ }^{32}$ conducted a numerical analysis of the evaporation process of small water droplets of diameters less than $1 \mathrm{~mm}$ on heated surfaces. They were the first to model the internal fluid motion due to thermocapillary convection in droplets. They found that fluid motion significantly affects the thermal field in the droplet and concluded that a model without fluid motion underpredicts the evaporation rates.

The flow field under the combined influence of surface tension and buoyancy effects has been referred to as buoyant-thermocapillary flow. These effects have been primarily studied with respect to flat liquid surfaces in floating zones, welding and glass manufacture. Kirdyashkin ${ }^{33}$ provided analytical solutions for velocity and temperature profiles in buoyant-thermocapillary driven flow in a 2D slot, and validated the solutions against experiments. Villers and Platten ${ }^{34}$ observed a single steady convection cell transition to a multicellular pattern with an increase in Ma. Numerical studies $^{35,36}$ demonstrated the importance of thermocapillary and combined buoyant-thermocapillary convection in rectangular containers heated from the side. It was shown that the convection mechanism augments the heat transfer by a factor of $2-2.5$.

Buffone et al. ${ }^{37}$ recently obtained $\mu$ PIV measurements to investigate thermocapillary convection with buoyancy effects near an evaporating meniscus in horizontally oriented glass capillaries of diameters ranging from 600 to $1630 \mu \mathrm{m}$ inner diameter (ID). Their measurements included flow patterns along horizontal and vertical diametrical cross sections near a meniscus pinned at the tube end. Symmetrical counter-rotating vortices were observed in the horizontal section due to thermocapillarity, while a single large vortex was seen to be induced by buoyancy in the vertical section; periodic instabilities were noted in both planes. However, their experimental setup introduced optical distortions because of the curvature of the glass tube, rendering measurements near the wall difficult to obtain. As only $70 \%$ of the test section was imaged, a comprehensive description of the flow profile could not be obtained.

The present work investigates steady buoyantthermocapillary convection induced by differential evaporation near a meniscus in horizontally oriented capillaries of sizes varying over a wide range from 75 to $1575 \mu \mathrm{m}$ ID. The problem under consideration is more complex than those discussed above due to the curvature of the free liquid surface, combined with the phase change prevalent and the buoyancy effects in play. A quantitative investigation of the threedimensional (3D) convection patterns is conducted at submillimeter length scales; 3D resolution at this scale has not been achieved in past studies. $\mu$ PIV measurements made over multiple orthogonal measurement planes illustrate the flow behavior. An analytical model is developed for vapor diffusion from a meniscus that depicts the intensive mass transport near the contact line. A scaling analysis is conducted to delineate the temperature differences governing pure thermocapillary flow in the horizontal center plane and buoyancy flow in the vertical center plane. The importance of thermocapillary convection in two-phase systems is highlighted.

\section{EXPERIMENTAL APPARATUS AND PROCEDURE}

The complex flow field near an evaporating meniscus is investigated using three different experiments as described below. The experiments help determine the threedimensional flow field, tube size effects on buoyancy, and evaporation flow rates, respectively.

\section{A. $\mu$ PIV measurements in horizontal cross sections of tubes}

Epifluorescent micro-particle image velocimetry ${ }^{38}$ ( $\mu$ PIV) was employed to map the small-scale spatial flow fields near an evaporating meniscus. The $\mu$ PIV system consists of an inverted Nikon microscope (TE 200), a two-cavity frequency-doubled ND-YAG laser (New Wave Research, Inc.), and an interline transfer CCD camera (LaVision Inc., 


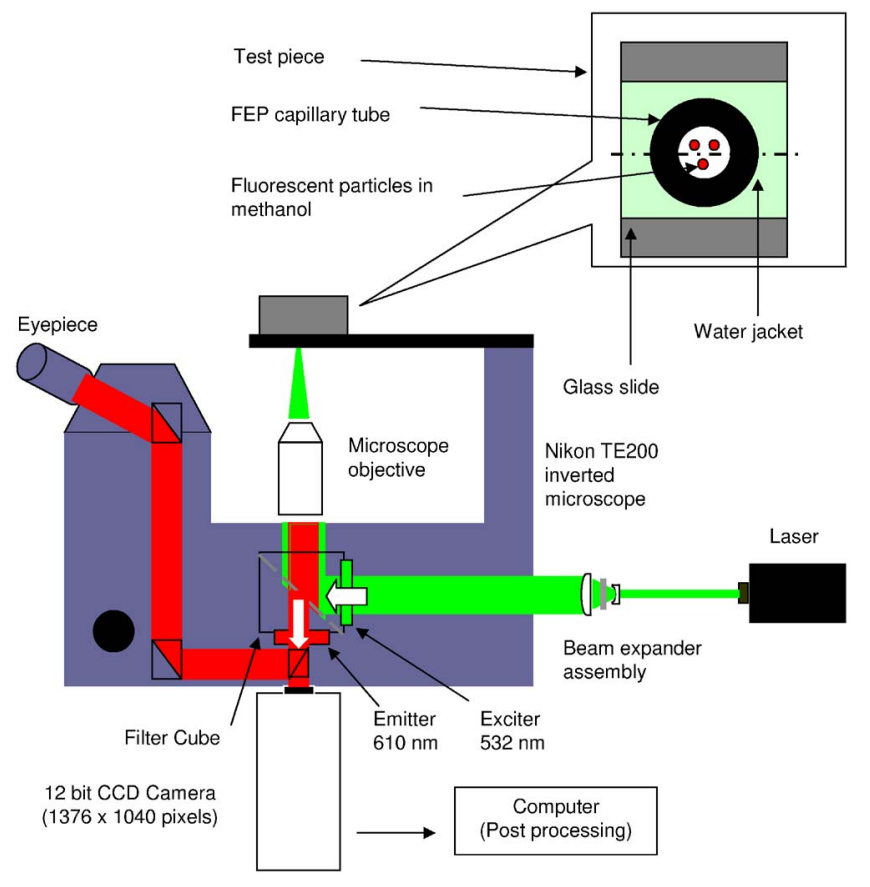

FIG. 1. (Color online) Experimental setup for horizontal $\mu$ PIV measurements.

Imager Intense) assembled on an optical table to minimize mechanical vibrations as shown in Fig. 1. The wavelength and pulse width of the laser beam are $532 \mathrm{~nm}$ and approximately $3-5$ ns, respectively. Full details of the measurement system can be found in Ref. 39 .

The test piece consists of a fluorinated ethylene propylene (FEP) capillary tube filled with methanol. The tube is surrounded by a water jacket enclosed between two glass slides and placed such that the tube axis is orthogonal to the direction of gravity. The similar refractive indices of FEP, methanol and water aid in minimization of optical distortions and allow for accurate velocity measurements to be obtained near the tube walls. The water bath surrounding the tube approximates an isothermal boundary condition on the walls. $70 \mathrm{~mm}$ long tubes are filled over two-thirds of their length with methanol such that both the menisci generated are approximately $10 \mathrm{~mm}$ away from the tube ends. Due to the micro-irregularities present on the tube surface, one of the menisci is pinned while the other recedes to compensate for the evaporation occurring at both the ends. Images for the current work are obtained at the stationary meniscus. All experiments are conducted at room temperature and under atmospheric pressure conditions. Five capillary tubes with inner diameters ranging from 75 to $1575 \mu \mathrm{m}$ are used to facilitate a study of the tube size effect on thermocapillary convection. Objective lenses of magnification $10 \times, 20 \times$, and $40 \times$ are used so as to provide the maximum possible resolution while still viewing the entire channel in the image.

Fluorescent polymer microspheres (Duke Scientific Co.) with a density of $1.05 \mathrm{~g} / \mathrm{cm}^{3}$ and diameter of $0.5 \mu \mathrm{m}$ are used to seed the fluid. These particles absorb green light $(\lambda$ $\sim 542 \mathrm{~nm})$ and emit red light $(\lambda \sim 612 \mathrm{~nm})$. A low particle concentration (volume fraction $\sim 200000: 1$ ) is used to avoid particle clumps. Their settling velocity is estimated to be
$4.2 \times 10^{-8} \mathrm{~m} \mathrm{~s}^{-1}$, which is very small in comparison to the mean fluid velocity of $10^{-4} \mathrm{~m} \mathrm{~s}^{-1}$. In addition, their sedimentation time for the smallest tube investigated $(75 \mu \mathrm{m})$ is estimated to be of the order of several hours, which is again negligible in comparison to the typical recording duration $(\sim 180 \mathrm{~s})$. The images are captured by the CCD camera with a time interval of $20 \mathrm{~ms}$ between successive images. The images are analyzed using EDPIV software. ${ }^{40}$

\section{B. Flow visualization in vertical center plane of tubes}

In order to investigate the effects of buoyancy, flow visualization is conducted at the vertical center planes of the tubes of different sizes considered above. This is achieved by placing a $45^{\circ}$ mirror between the lens and the camera as described in Ref. 39. These experiments are conducted using an upright Nikon Eclipse (ME600) microscope with an interline transfer CCD camera (Roper Scientific Photometrics, CoolSNAP HQ). The devices were controlled using Metamorph imaging software (Universal Imaging Corporation). A Nikon mercury arc lamp is used as the illumination source. Long-exposure $(\sim 30 \mathrm{~ms})$ streak images are obtained at a time interval of $0.3 \mathrm{~s}$ between successive images. Since very low particle concentrations are used, several images are superposed to obtain a clearer depiction of the flow field.

\section{C. $\mu$ PIV measurements of evaporative flow rate}

The objective of this experiment is to measure the evaporative mass flow rates as a function of tube size. The test piece used is a variant of that used for the horizontal cross sections discussed in Sec. II A. Here, one end of the capillary tube is placed in a reservoir of fluid such that there is a continuous liquid supply to the evaporating meniscus pinned at the other end. The rest of the test setup is the same as that described in Sec. II A. Images taken far from the meniscus are interrogated to obtain bulk velocity measurements.

\section{EXPERIMENTAL RESULTS}

The three-dimensional flow field in a capillary tube of $400 \mu \mathrm{m}$ diameter is first considered in detail. Tube-size effects on thermocapillarity, buoyancy, and evaporative flow rates are then discussed.

\section{A. 3D flow pattern}

To understand the three-dimensionality of the convective flow pattern, $\mu$ PIV measurements were made along three horizontal planes at varying depths as identified in Fig. 2 near the evaporating meniscus in a $400 \mu \mathrm{m}$ tube. Figures 3 and 4 depict vector maps of the flow field. Only a subset of the planar velocity vectors $(\mathbf{V})$ obtained are shown to improve clarity. The magnitude of vorticity $(\omega)$ calculated using the relation $\omega=\boldsymbol{\nabla} \times \mathrm{V}$ is represented as contours in these figures. Central-order differencing was used at the interior points and a forward-differencing scheme was used at the boundaries (walls and meniscus) to evaluate the vorticity magnitude. The spatial resolutions of the differencing 


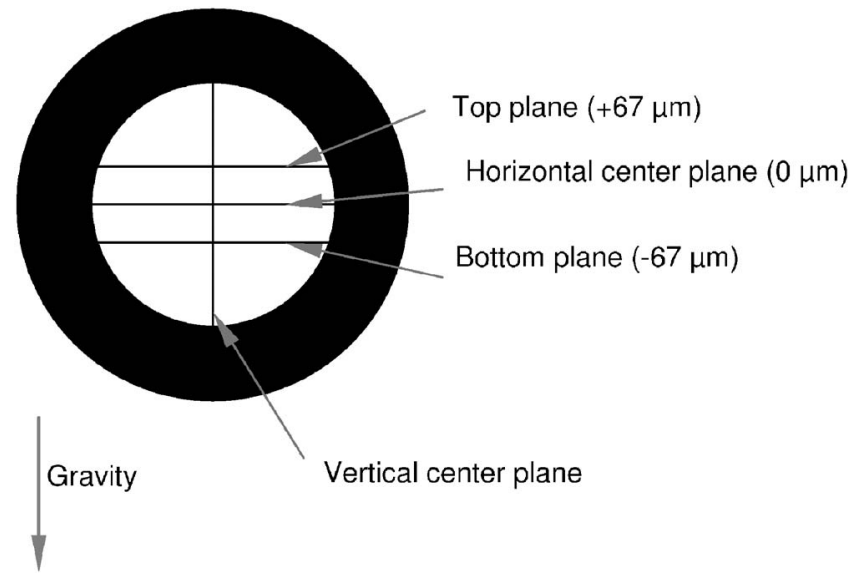

FIG. 2. Schematic of $400 \mu \mathrm{m}$ tube cross section showing the location of measurement planes.

scheme were 4, 5, 10, 21, and $21 \mu \mathrm{m}$ for 75, 200, 400, 762, and $1575 \mu \mathrm{m}$ tubes, respectively. Streamtraces are also superposed on the figures to readily illustrate the flow profile.

Figure 3 details the velocity field near the meniscus along the horizontal measurement planes. In Fig. 3(a), the flow is directed towards the meniscus; two small counterrotating vortices are observed at the corners. These vortices are observed to grow in size and strength to form an axisymmetric flow pattern at the horizontal center plane [Fig. 3(b)]. In Fig. 3(c), the flow is directed uniformly away from the meniscus and shows no vortices. The flow field at the different horizontal planes thus indicates the existence of a symmetric counter-rotating vortex pair that grows from the top plane to the center plane and vanishes in the lower half of the tube cross section. Though the top and bottom planes are equally spaced from the horizontal center plane, a flow towards the meniscus at the top planes and away from the meniscus at the bottom planes indicates a clear asymmetry in the flow along the vertical center plane. This asymmetry along the vertical center plane is further clarified later in the paper, in Fig. 5(c), where a single counterclockwise vortex is shown to dominate the flow and push the other vortex to a corner. This small clockwise vortex forms the beginning of the vortex tube that grows in size (symmetrically with respect to the vertical axis) to form symmetrical counterrotating vortices in the horizontal top [Fig. 3(a)] and center planes [Fig. 3(b)] and a large vortex in the vertical center plane [Fig. 5(c)]. This analysis suggests the presence of a closed vortex tube in the form of an asymmetric toroid near the meniscus. Additional images from this flow field at eight different measurement planes are available in a previous publication. $^{41}$

\section{B. Tube-size effects \\ 1. Thermocapillarity}

Figure 4 shows the flow fields near the evaporating meniscus along the horizontal center plane for all five tube diameters investigated: 75, 200, 400, 762, and $1575 \mu \mathrm{m}$. It may be noted that in Fig. 4(e), roughly a half of the $1575 \mu \mathrm{m}$ tube is imaged because of the restriction imposed by the field of view of the objective lens. However, the flow field was visually observed to be axisymmetric. The flow fields are seen to be similar for all five tubes and include two counterrotating symmetrical vortices; only one vortex is seen for the largest tube since only half the plane could be included in the measurements for this tube diameter. It is interesting to note that the vortex centers are at the same nondimensional radial location irrespective of tube size. While the velocity vectors right at the meniscus surface could not be measured for the $75 \mu \mathrm{m}$ diameter tube due to difficulties with spatial resolution, the profiles for the other tube sizes reveal the location of highest vorticity magnitude to be along the meniscus. This indicates that the sharpest velocity gradients are present along the meniscus. In addition, the maximum velocity location is observed to be at the interface, as is expected of purely thermocapillary-driven flow, and is midway between the center of the meniscus and the contact line [seen clearly in Fig. 4(e)].

The temperature gradient induced due to the differential evaporative cooling along the meniscus is proposed to be the cause for the observed flow pattern along the horizontal cen-

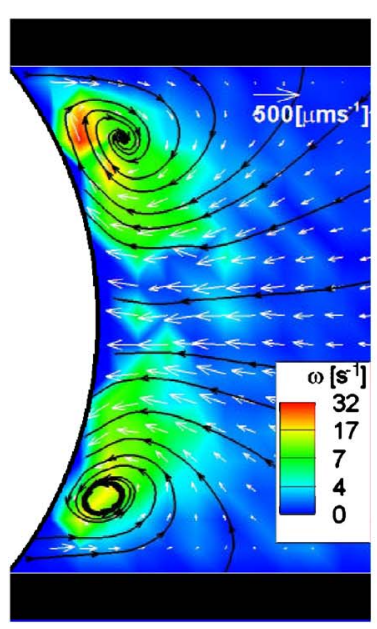

(a)

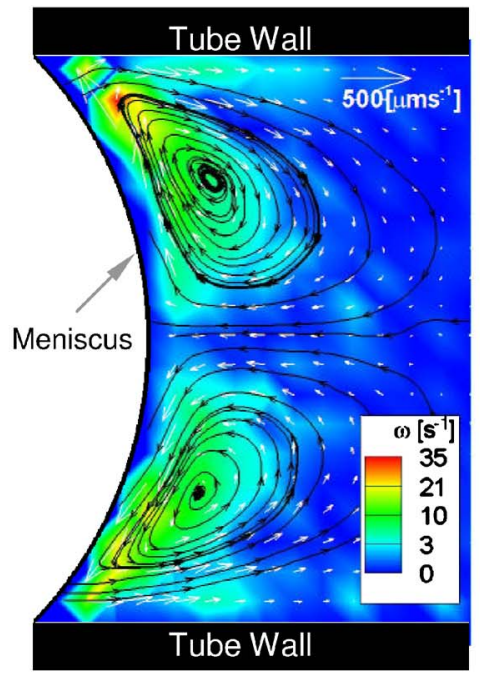

(b)

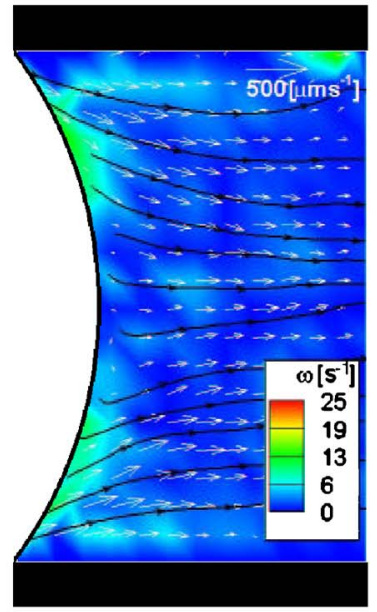

(c)
FIG. 3. (Color online) Velocity vectors with vorticity magnitude contours in $400 \mu \mathrm{m}$ tube at different measurement planes (a) top plane, (b) horizontal center plane, and (c) bottom plane, as identified in Fig. 2. 


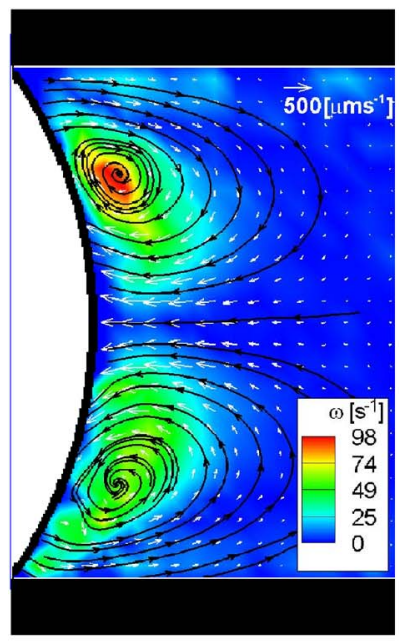

(a) $75 \mu \mathrm{m}$

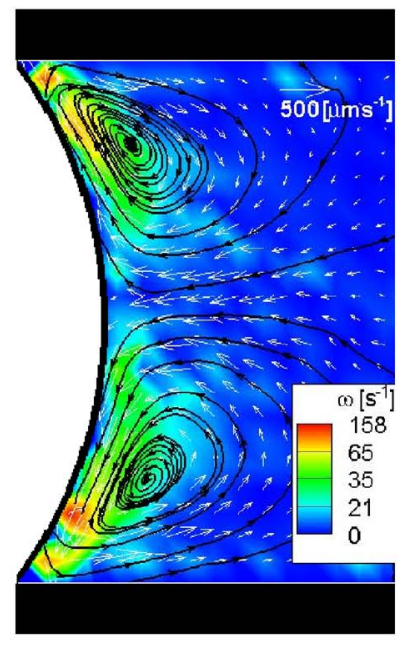

(b) $200 \mu \mathrm{m}$

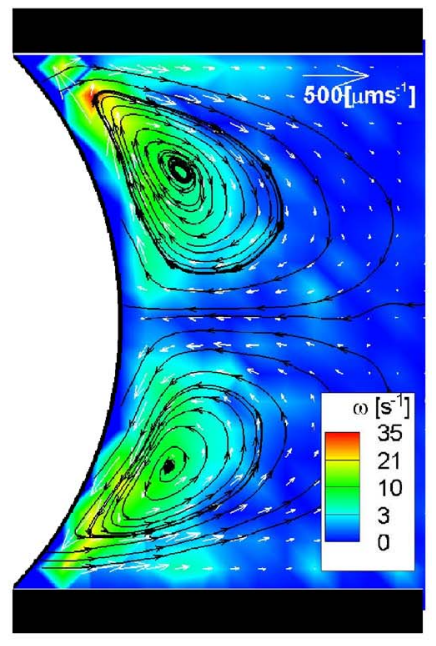

(c) $400 \mu \mathrm{m}$

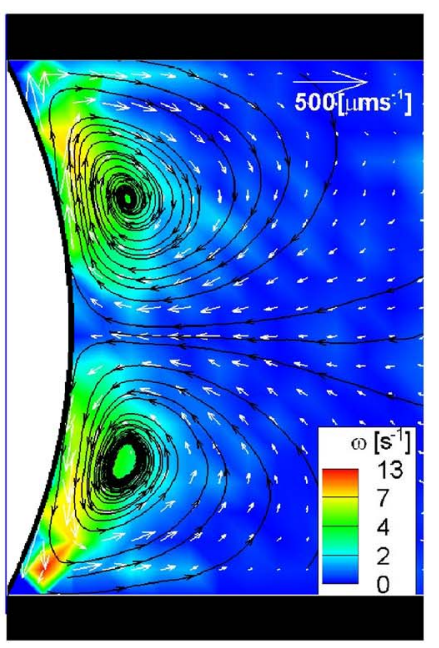

(d) $762 \mu \mathrm{m}$

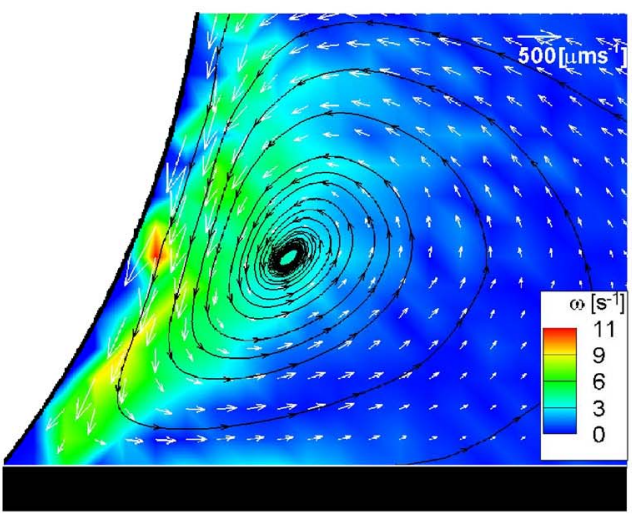

(e) $1575 \mu \mathrm{m}$
FIG. 4. (Color online) Velocity vectors with vorticity contours obtained at horizontal center planes for different tubes. In (e), only one-half of the meniscus region is imaged. ter planes in Fig. 4. Because of the intensive evaporation near the corners, the local liquid-vapor interface temperature is less than that at the meniscus center. Since for methanol (and for most liquids), surface tension decreases with an in- crease in temperature, the surface tension is higher near the corners than at the center. This drives flow towards the corners, establishing the two counter-rotating symmetrical vortices observed at the horizontal center plane for all the tube

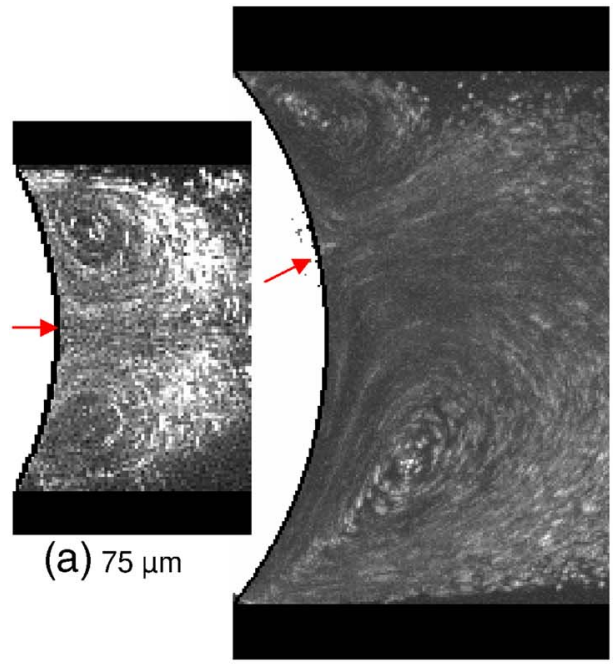

(b) $200 \mu \mathrm{m}$

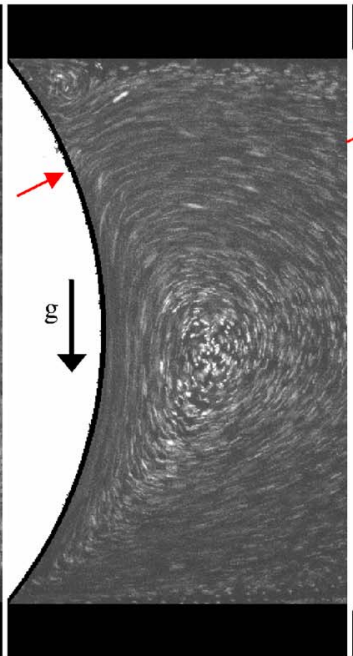

(c) $400 \mu \mathrm{m}$

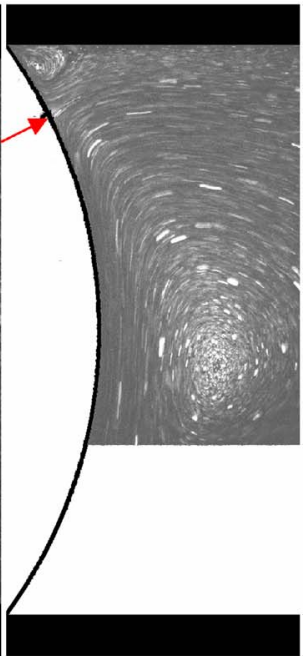

(d) $762 \mu \mathrm{m}$
FIG. 5. (Color online) Long-exposure particle streak images at the vertical center planes for different tubes. Multiple images were superimposed to improve contrast. In (d), only $70 \%$ of the tube is imaged ( $g$ is gravitational acceleration). 
sizes. These symmetrical vortices are in qualitative agreement with past experimental ${ }^{37}$ and numerical work ${ }^{22,42}$ involving evaporation of liquids from microcapillaries.

\section{Buoyancy}

Since the tube is oriented with its axis being horizontal in the present experiments, buoyancy effects are found to affect the flow pattern near the meniscus; the observed asymmetrical flow (Fig. 5) is the combined result of buoyancy and thermocapillarity effects. The relative influences of buoyancy and thermocapillarity on the flow were characterized with long-exposure particle streak images obtained along the vertical center plane for different tube sizes, as shown in Fig. 5; multiple images are superimposed for each part of the figure to improve contrast. The arrows in the figure point to the location of the stagnation point on the meniscus.

As evaporation occurs at the interface, it renders the meniscus region relatively cooler than the bulk liquid away from the meniscus. This difference in temperature gives rise to buoyancy effects which grow in strength with tube diameter. For the smallest diameter investigated $(75 \mu \mathrm{m})$, buoyancy effects are negligible and the same symmetrical flow pattern is observed [Fig. 5(a)] as seen in the corresponding horizontal center-plane velocity map [Fig. 3(a)]; this indicates a donut-shaped vortex tube near the meniscus. For the $200 \mu \mathrm{m}$ tube, asymmetry sets in and the counterclockwise bottom vortex is found to be larger than the clockwise upper one indicating the presence of an asymmetrical toroidal vortex as discussed earlier. As buoyancy effects increase with length scale, the counterclockwise vortex grows in size with an increase in tube size as seen in Figs. 5(c) and 5(d). The stagnation point is seen to move upwards along the meniscus with an increase in buoyancy. It may be seen that the flow is divided into two regions: an upper region where the motion is induced by surface tension and a lower one driven by density differences. The increase in size of the buoyancydriven vortex with length scale was also reported in Ref. 43. The degree of cooling produced at the interface (which drives the buoyancy-induced flow) is dependent on the mass flux.

\section{Evaporative flow rate}

The evaporative mass fluxes and flow rates are obtained from the experiments described in Sec. II C as a function of tube diameter. The fully developed parabolic flow profiles obtained (results not shown) from the $\mu$ PIV analysis are integrated over the cross-sectional area to obtain the mean flow velocity. This mean velocity is used to estimate the mass flow rates and mass fluxes. These mass flow rates $\dot{m}$ and the corresponding mass fluxes $\dot{m}^{\prime \prime}$ are plotted as a function of tube size $(D)$ in Fig. 6.

The evaporative mass flow rate increases with tube diameter because of the larger available interfacial area, whereas the flux is seen to decrease with an increase in diameter. These higher mass fluxes in smaller tubes drive stronger recirculation patterns (represented by vorticity) as seen in Fig. 7. The vorticity at the vortex center $\omega_{t}$ as a function of tube size is plotted in this figure. This inverse

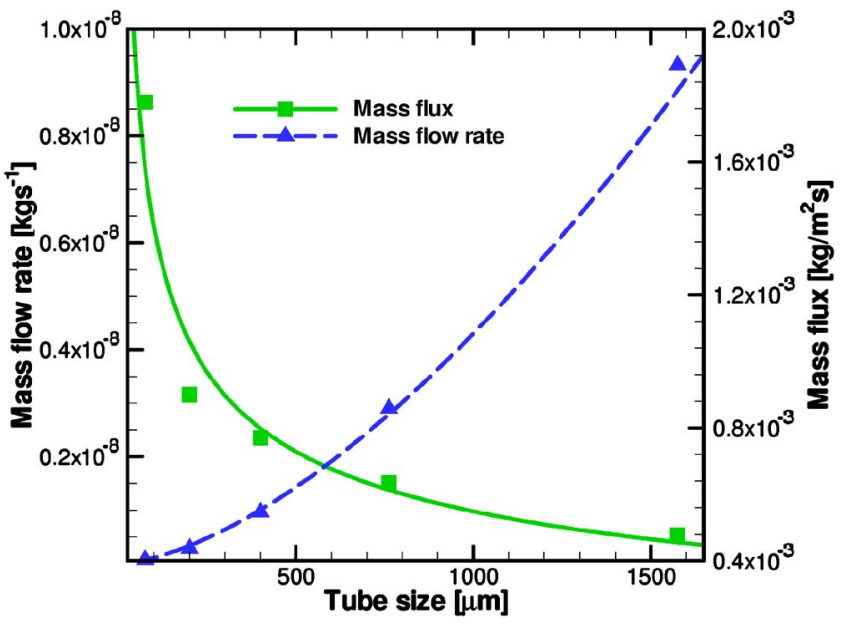

FIG. 6. (Color online) Mass flow rate and mass flux as a function of tube size.

hyperbolic dependence of vorticity on tube diameter is in agreement with previous numerical work, ${ }^{42}$ but is in contrast to the linear relationship obtained in Ref. 37; however, only three data points were used to suggest this linear relationship.

Power-law curve fits to the mass flow rate and mass flux results yield $\dot{m} \propto D^{1.5}$ and $\dot{m}^{\prime \prime} \propto D^{-0.5}$. If the evaporation were uniformly distributed over a surface, the evaporation rate would be proportional to the area of the interface $\left(\propto D^{2}\right)$. If instead, evaporation took place only over the thin-film region, the evaporation rate would be proportional to the perimeter of the meniscus $(\propto D)$. The diameter exponent of 1.5 suggested by the results lies in between these two bounds, supporting the more probable case of differential evaporation. Such behavior has been observed earlier by Peiss, ${ }^{44}$ who measured the evaporation of small water droplets of diameter ranging from 0.046 to $0.36 \mathrm{~cm}$ maintained at constant volume. He observed that the evaporation rate is not proportional to droplet diameter or surface area, but instead lay between the two bounds; however, an exponent for the

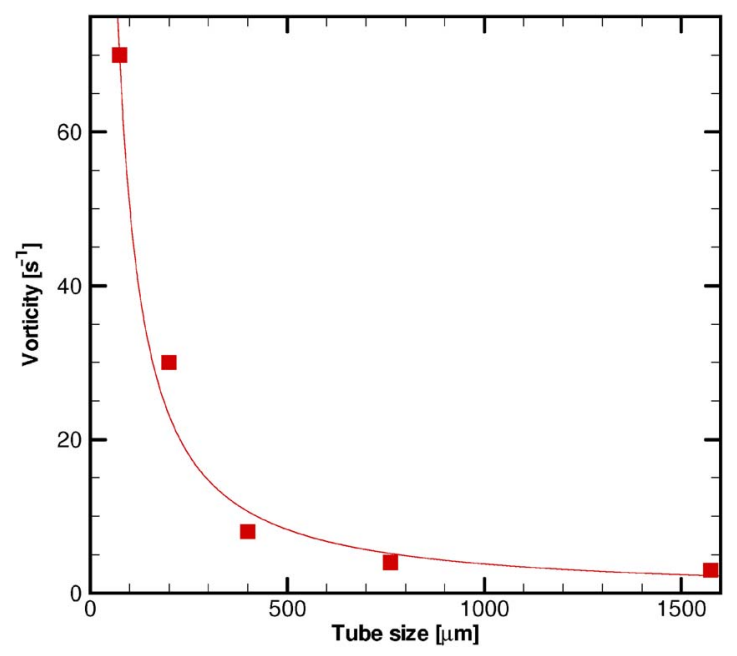

FIG. 7. (Color online) Variation with tube size of the vorticity at the vortex center in horizontal center-plane vector maps. 
diameter dependence was not specified. Hisatake et $a l .{ }^{45}$ also measured evaporation rates of water in circular vessels open to the atmosphere and observed an evaporation-rate dependence of $D^{1.8}$. However, recent experimental ${ }^{46}$ and numerical ${ }^{42}$ work involving evaporation of methanol in three tubes of diameters 200, 600, and $900 \mu \mathrm{m}$ demonstrated a linear relationship between evaporation rate and tube size. Further research is necessary to understand the dependence of evaporation rate on tube size.

\section{MODELING}

\section{A. Analytical model for vapor diffusion from a meniscus}

The underlying physics of differential evaporation at a meniscus is now discussed. The evaporation of a volatile liquid from a stationary meniscus into atmosphere at room temperature is primarily governed by Fick's law of binary diffusion. The air near the liquid surface becomes saturated with methanol vapor; due to the lower far-field vapor concentration, the vapor diffuses away from the meniscus. This continuous vapor diffusion process is represented by a steady-state solution to the Laplace equation, i.e., $\nabla^{2} \phi=0$, with boundary conditions of saturated vapor $\phi_{\text {sat }}$ at the liquid surface and $\phi_{\infty}$ at infinity. The local evaporative heat flux $q^{\prime \prime}(r)$ on the meniscus as a function of distance from the axis $r$ can be written as $q^{\prime \prime}(r)=-\left.\rho \gamma h_{f g} \boldsymbol{\nabla} \phi\right|_{l v}$, where $\gamma$ is the methanol-air diffusion coefficient and $\left.\boldsymbol{\nabla} \phi\right|_{l v}$ is the vapor concentration gradient at the liquid-vapor interface.

The solution methodology is motivated by a similar problem considered by Deegan et al. ${ }^{47}$ They derived an analytical solution for the vapor concentration profile near a saturated drop on an impermeable substrate by considering an equivalent electrostatic problem of a lens-shaped conductor in free space.

An inverse problem is solved in the present work, where the meniscus is approximated as a spherical cap of radius $R_{0}$ and the atmosphere as an infinitely large spherical surface. The region bounded between these two intersecting spheres forms the domain of the problem. The contact angle $\theta_{c}$ of methanol in FEP capillary tubes is determined to be $50^{\circ} \pm 3^{\circ}$ from images of the meniscus as shown in Ref. 40. With $\phi_{\text {sat }}=0.147, \phi_{\infty}=0$, and $\theta_{c}=50^{\circ}$ as inputs, the exact solution to the local evaporative heat flux is given by Eq. (A3) developed in the Appendix, and is closely approximated as

$$
q^{\prime \prime}(r)=0.0516\left[1-(r / R)^{2}\right]^{-0.91} \rho \gamma h_{f g} / R .
$$

Equation (1) closely resembles the solution for a droplet obtained by Deegan et al. ${ }^{48}$ and given by $q^{\prime \prime}(r) \propto\left[1-(r / R)^{2}\right]^{-\lambda}$, where $r$ is the radial distance from the axis, $R$ is the radius of the drop, and $\lambda=\left(\pi-2 \theta_{c}\right) /\left(2 \pi-2 \theta_{c}\right)$. An inverse dependence of heat flux on the tube size is reflected in Eq. (1) and conforms with the mass flow rate results obtained experimentally (Fig. 6).

To avoid singularity at the contact line, a value of $r / R$ $=0.999$ is chosen to evaluate the heat flux from Eq. (1). Figure 8 shows the dependence of dimensionless heat transfer and heat flux on the dimensionless radial position along the interface. The cumulative heat transfer rate $q_{R-r}$ repre-

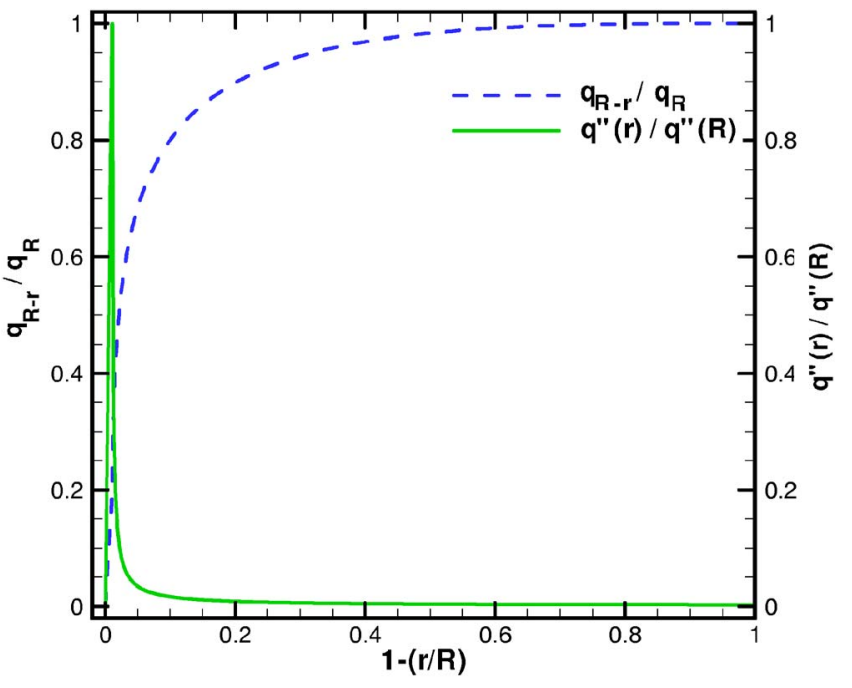

FIG. 8. (Color online) Dimensionless heat transfer (left) and heat flux (right) along the meniscus obtained from the analytical model.

sents the heat transfer rate from a zone along the meniscus extending from the contact line $r=R$ to a location $r=r ; q_{R}$ thus represents the total heat transfer rate from the meniscus. The Laplace solution yields a higher gradient of vapor fraction at the corners than at the axis because of the curvature of the meniscus. This leads to higher mass fluxes and correspondingly higher heat fluxes at the corners, contributing to the bulk of the heat transfer from the meniscus. Almost 95\% of the total heat transfer takes place from $30 \%$ of the meniscus region that is close to the contact line. The heat flux trend is similar to that found in previous numerical results. ${ }^{42,43}$ It is this differential evaporation that generates a temperature gradient causing the surface flow. The dimensional independence of the solution for the local heat flux along the interface reflected in its nondimensional form is also confirmed in the horizontal center-plane vector maps which showed that vortex center occurs at the same nondimensional location irrespective of the tube size.

\section{B. Scaling analysis}

As discussed earlier, the temperature difference along the meniscus because of differential evaporation governs the thermocapillary convection in the horizontal center plane, while the temperature difference between the bulk and the cooler interface governs the buoyancy-driven flow in the vertical center plane. While it is desirable to obtain the local interface temperatures along the meniscus, direct local measurements of temperature at such small scales is challenging. Some efforts have been made in the literature towards such measurements, using thermocouples ${ }^{49}$ and infrared measurements. ${ }^{50}$ Alternatively, a characteristic temperature difference that drives the thermocapillary flow can be inferred from the experimental velocities by a scaling analysis.

Following the approach of Ref. 31, a spherical coordinate system is used with origin at the center of the meniscus (see Fig. 9), and a shear stress balance at the interface is performed. A first-order series expansion of $v_{\theta} / r$ at a radial 


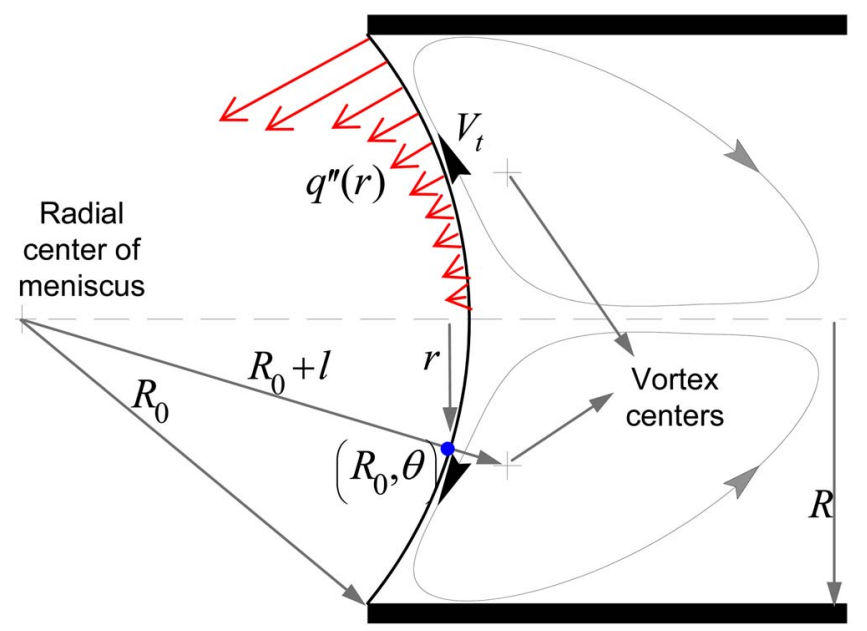

FIG. 9. (Color online) Schematic representation of thermocapillary vortices at the horizontal center plane showing the heat fluxes $q^{\prime \prime}(r)$ along the meniscus and the location of maximum velocity $V_{t}$ indicated as a solid dot at $\left(R_{0}, \theta\right)$.

position near the interface $R_{0} \leq r \leq R_{0}+l$, where $R_{0}+l$ denotes the radial location of the vortex center at which the velocity is zero, leads to

$$
v_{\theta}\left(R_{0}, \theta\right)=(d \sigma / d T)\left(\partial T /\left.\partial \theta\right|_{\left(R_{0}, \theta\right)}\right) \ln \left[1+\left(l / R_{0}\right)\right] / \mu,
$$

where $v_{\theta}\left(R_{0}, \theta\right)$ and $\partial T /\left.\partial \theta\right|_{\left(R_{0}, \theta\right)}$ represent the tangential velocity and interfacial temperature gradient, respectively. The point $\left(R_{0}, \theta\right)$ (Fig. 9) on the interface is located on a radial line from the center of spherical cap to the vortex center. $\mu$ and $d \sigma / d T$ denote the viscosity and temperature coefficient of surface tension of the liquid, respectively. The point $\left(R_{0}, \theta\right)$ also signifies the location of maximum velocity along the interface [as seen in Fig. 4(d) and schematically represented in Fig. 9]. However, the maximum velocity data obtained from $\mu \mathrm{PIV}$ measurements is not employed because of the lack of interface velocity measurements for the $75 \mu \mathrm{m}$ tube. Instead, this velocity $v_{\theta}\left(R_{0}, \theta\right)$ is estimated from the vorticity at the vortex center $\omega_{t}$. Using the relation $\omega=\nabla$ $\times \mathrm{V}$ and some simplifications employed in deriving Eq. (2), the following relation is obtained:

$$
v_{\theta}\left(R_{0}, \theta\right)=\omega_{t}\left[l+\left(l^{2} / 2 R_{0}\right)\right] .
$$

Since the dimensionless location $l / R_{0}$ of the vortex center is a constant, it can be deduced from Eq. (3) that $\omega_{t} \propto l^{-1}$ $\propto R^{-1}$, that is, vorticity $\omega_{t}$ is inversely proportional to tube diameter, which is also observed in Fig. 7.

Scaling $\partial T /\left.\partial \theta\right|_{l v}$ as $\Delta T_{t}$ and $v_{\theta}$ as $V_{t}$, the characteristic temperature difference and velocity of thermocapillary flow, respectively, it follows from Eqs. (2) and (3) that

$$
\Delta T_{t}=\mu \omega_{t}\left[l+\left(l^{2} / 2 R_{0}\right)\right] /(d \sigma / d T) \ln \left[1+\left(l / R_{0}\right)\right] .
$$

For the $400 \mu \mathrm{m}$ tube, with $\omega_{t}=8 \mathrm{~s}^{-1}, l=40 \mu \mathrm{m}$, and properties listed in Table $\mathrm{I}$, a $\Delta T_{t}$ of $30 \mathrm{mK}$ is obtained.

In terms of the temperature difference driving the flow in the vertical center plane, the evaporation heat flux cools the interface and sets up a temperature difference between the bulk liquid and the interface. This temperature gradient is believed to govern the buoyancy-driven convection. Assum-
TABLE I. Fluid properties ( $298 \mathrm{~K}, 1 \mathrm{~atm})$.

\begin{tabular}{lc}
\hline \hline \multicolumn{1}{c}{ Fluid } & Methanol \\
\hline$\mu(\mathrm{kg} / \mathrm{m} \mathrm{s})$ & $8.56 \times 10^{-4}$ \\
$d \sigma / d T(\mathrm{~N} / \mathrm{m} \mathrm{K})$ & $-8.0 \times 10^{-5}$ \\
$\chi(1 / \mathrm{K})$ & $1.2 \times 10^{-3}$ \\
$\kappa\left(\mathrm{m}^{2} / \mathrm{s}\right)$ & $1.75 \times 10^{-7}$ \\
$\rho\left(\mathrm{kg} / \mathrm{m}^{3}\right)$ & 786 \\
\hline \hline
\end{tabular}

ing buoyancy to be the dominant mechanism, a characteristic velocity of this flow $V_{b}$ can be written as $V_{b}=\sqrt{g \chi \Delta T_{b} D}$, where $g, \chi$, and $\Delta T_{b}$ denote the acceleration due to gravity, the volumetric thermal expansion coefficient of the liquid, and the characteristic temperature difference for buoyancydriven flow, respectively. For the $400 \mu \mathrm{m}$ tube, choosing the maximum velocity of $1 \mathrm{~mm} / \mathrm{s}$ (see Ref. 41) as $V_{b}$ gives a $\Delta T_{b}$ of $0.21 \mathrm{~K}$, which is an order of magnitude higher than the $\Delta T_{t}$ obtained above for thermocapillary flow along the meniscus. This difference in characteristic temperatures governing the flow in the horizontal and vertical center planes was not noted in earlier studies. ${ }^{37}$

In the absence of gravity, a symmetrical thermocapillary flow would be expected even in the vertical center plane. But under terrestrial conditions, buoyancy-driven flow is superposed on the thermocapillary flow. As the buoyancy force is a bulk phenomenon while the thermocapillary force is a surface phenomenon, the ratio of $\mathrm{Gr} / \mathrm{Re}^{2}$ characterizing the competing buoyancy and inertial forces (driven only by the thermocapillary force) governs the system. The nondimensional parameters are defined as $\mathrm{Gr}=\rho^{2} g \chi \Delta T_{b} D^{3} / \mu^{2}$ and $\mathrm{Re}=\rho V_{t} D / \mu$. The $\mathrm{Gr} / \mathrm{Re}^{2}$ ratio for the $400 \mu \mathrm{m}$ tube yields a value of 9 , confirming the dominance of buoyancy forces for this tube size seen in the experiments. Since $\mathrm{Gr} / \mathrm{Re}^{2} \propto D$, an increase in this length scale enhances the buoyancy effects. This is also observed in the flow visualization experiment results (Fig. 5), which show the increase in size of the buoyancy-driven vortex with increase in tube size.

Similar results were observed in a floating zone by Kobayashi, ${ }^{51}$ who found buoyancy-driven convection to be dominant over Marangoni convection when the zone length was greater than a critical value. Similarly, in a combined convection problem of a liquid layer in a cube subjected to different temperatures at the walls, an increase in Rayleigh number suppressed the thermocapillary vortex. ${ }^{35}$

With $V_{t}$ as the characteristic velocity, a Marangoni number can be defined for the curved geometry ${ }^{52}$ as Ma $=V_{t} D / \kappa$, where $\kappa$ denotes the thermal diffusivity of liquid. This is identical to the Péclet number, which captures the convective transport of energy in the liquid domain. $V_{t}$ is evaluated from Eq. (3) with $\omega_{t}$ obtained from Fig. 7. The Ma values for different tubes are listed in Table II. The values, of the order of unity, indicate that thermocapillarity significantly affects the thermal field in the system in contrast to a conduction-only case. These mixing effects increase the heat transfer as discussed in Sec. I. The Marangoni number is seen to increase with an increase in tube size. This suggests an enhanced role of convection effects in augmenting heat 
TABLE II. Values of Marangoni number for various tube sizes.

\begin{tabular}{cc}
\hline \hline $\begin{array}{c}\text { Tube size } \\
(\mu \mathrm{m})\end{array}$ & $\begin{array}{c}\text { Marangoni } \\
\text { number }\end{array}$ \\
75 & 0.2 \\
200 & 0.6 \\
400 & 0.8 \\
762 & 1.6 \\
1575 & 3.6 \\
\hline \hline
\end{tabular}

transfer from large tubes. However, this result contradicts Ref. 37, in which a decrease in Ma was observed with an increase in tube size. This apparent contradiction is attributable to their definition of Marangoni number $\mathrm{Ma}^{\prime}$, defined as $\mathrm{Ma}^{\prime}=(d \sigma / d T)(\partial T / \partial x) x^{2} /(\mu \kappa)$, which was based on the experimentally measured temperature gradient $(\partial T / \partial x)$ driving the system and a length scale $x$. A fixed length scale $x$ of $50 \mu \mathrm{m}$ was used although the tube sizes examined were in the range of 600 to $1630 \mu \mathrm{m}$. In the present work, as no temperature measurements are made, a Marangoni number based on flow velocity obtained from PIV analysis is used instead as described.

In two-phase heat transfer systems, greater temperature differences exist when heating is present, in contrast to the room temperature conditions under which vapor diffusion dominates. The wall superheats present in such systems would induce much higher temperature gradients along the meniscus than those observed in the present experiments $(\sim 30 \mathrm{mK})$. This proportionately increases the surface tension gradients which in turn drive stronger vortices. The flow field is also rendered more complex due to the strong gravitational effects. Vigorous circulation would enhance heat transfer but could also prove detrimental by causing instabilities leading to dryouts and ruptures observed in falling films, ${ }^{10}$ liquid layers, ${ }^{53}$ and liquid bridges. ${ }^{11}$ Thus, thermocapillary convection becomes much more critical in twophase systems.

\section{CONCLUSIONS}

Microfluidic visualization techniques were employed to quantitatively map the $3 \mathrm{D}$ nature of steady convection patterns near an evaporating meniscus in horizontally oriented microcapillaries. $\mu$ PIV was used to make quantitative velocity and vorticity measurements, while long-exposure streak images were used to observe the qualitative flow pattern in the vertical center planes. In the horizontal center plane, two counter-rotating symmetrical vortices were observed, whereas in the vertical center plane, a single large vortex was observed. For tests conducted in tubes of different diameters, the horizontal planes revealed a stronger counter-rotating symmetrical vortex pair in smaller tubes, whereas the vertical center planes revealed a larger single vortex in larger tubes. It is concluded that for an evaporating meniscus in capillary tubes and for a given set of experimental conditions, a critical diameter exists such that for smaller diameters, the flow is driven primarily by thermocapillarity. Buoyancy effects set in beyond this critical diameter leading to three-dimensionalities in the flow. The evaporative mass flow rates were found to show a dependence on tube diameter of $D^{1.5}$. An analytical model is presented which supports the importance of the thin-film region even in vapordiffusion-dominated evaporative processes. A scaling analysis was performed to delineate the significance of thermocapillary convection.

In ongoing work, microscale temperature measurements coupled with flow visualization are being performed to provide a more comprehensive understanding of the problem.

\section{ACKNOWLEDGMENTS}

The authors acknowledge the financial support of the members of the Cooling Technologies Research Center, a National Science Foundation Industry/University Cooperative Research Center. Professor Steve Wereley's insightful help with the $\mu \mathrm{PIV}$ measurements is appreciated.

\section{APPENDIX: SOLUTION TO THE EVAPORATING MENISCUS BOUNDARY VALUE PROBLEM}

A spherical cap at a constant potential is considered in a semi-infinite space at zero potential. The solution of the Laplace equation for this geometry is sought from an equivalent boundary-value problem that is solved using spherical harmonics. A toroidal coordinate system is used to solve the interior Dirichlet problem in the domain bounded by a spherical cap (meniscus) and an infinitely large sphere. A general solution to the electrostatic potential in a problem of intersecting spheres is given in Ref. 54:

$$
\begin{aligned}
\phi(\alpha, \beta)= & \sqrt{2(\cosh \alpha-\cos \beta)} \\
& \times \int_{0}^{\infty} \frac{\phi_{\text {sat }} \cosh \left(\pi-\beta_{2}\right) \tau}{\cosh \pi \tau} \frac{\sinh \left(\beta-\beta_{1}\right) \tau}{\sinh \left(\beta_{2}-\beta_{1}\right) \tau} \\
& \times P_{-1 / 2+i \tau}(\cosh \alpha) d \tau,
\end{aligned}
$$

where the toroidal coordinates $\alpha$ and $\beta$ represent the toroidal and spherical surfaces, respectively, $P_{-1 / 2+i \tau}(\cosh \alpha)$ are the Legendre functions of the first kind, and $\beta_{2}=(3 \pi / 2)-\theta_{c}$ and $\beta_{1} \rightarrow 0$ indicate the meniscus and infinitely large spherical surface, respectively. A relationship between the toroidal coordinate $\alpha$ and cylindrical coordinate $r$ is arrived at from geometrical considerations as

$$
\alpha=\cosh ^{-1}\left[\cos \beta_{2}+\frac{\sin \beta_{2}}{\tan \theta_{c}-\sqrt{\sec ^{2} \theta_{c}-(r / R)^{2}}}\right] .
$$

The local evaporative heat flux at the interface is calculated from $q^{\prime \prime}(r)=-\rho \gamma h_{f g} \nabla \phi$ at the meniscus: 


$$
\begin{aligned}
q^{\prime \prime}(r) & =-\left.\frac{\rho \gamma h_{f g}}{R}\left(\cosh \alpha-\cos \beta_{2}\right)_{\partial_{\beta} \phi}\right|_{\beta=\beta_{2}} \\
& =-\frac{2 \sqrt{2} \rho \gamma h_{f g} \phi_{\text {sat }}}{\pi R}\left(\cosh \alpha-\cos \beta_{2}\right)^{3 / 2} \int_{0}^{\infty} \frac{\cosh \left(\pi-\beta_{2}\right) \tau}{\cosh \pi \tau}\left[\frac{\sin \beta_{2}}{2\left(\cosh \alpha-\cos \beta_{2}\right)}+\tau \tanh \beta_{2} \tau\right] P_{-1 / 2+i \tau}(\cosh \alpha) d \tau
\end{aligned}
$$

Equation (A3) was computed numerically and compared to the approximate form given in Eq. (2), showing excellent agreement.

${ }^{1} \mathrm{~J}$. Thomson, "On certain curious motions observable at the surfaces of wine and other alcoholic liquors," Philos. Mag. 10, 330 (1855).

${ }^{2} \mathrm{C}$. Marangoni, On the Expansion of a Drop of Liquid Floating on the Surface of Another Liquid (Tipographia dei Fratelli Fusi, Pavia, Italy, 1865).

${ }^{3} \mathrm{~A}$. V. Hershey, "Ridges in a liquid surface due to the temperature dependence of surface tension," Phys. Rev. 56, 204 (1939).

${ }^{4} \mathrm{H}$. Benard, "Les tourbillons cellulaires dans une nappe liquide," Rev. Gen. Sci. Pures Appl. 11, 1261 (1900).

${ }^{5} \mathrm{~L}$. Rayleigh, "On convective currents in a horizontal layer of fluid when the higher temperature is on the under side," Philos. Mag. 32, 529 (1916).

${ }^{6} \mathrm{M}$. J. Block, "Surface tension as the cause of Benard cells and surface deformation in a liquid film," Nature (London) 178, 650 (1956).

${ }^{7}$ V. G. Levich, Physicochemical Hydrodynamics (Prentice-Hall, Englewood Cliffs, NJ, 1962).

${ }^{8}$ J. R. A. Pearson, "On convection cells Induced by surface tension," J. Fluid Mech. 4, 489 (1958).

${ }^{9}$ L. E. Scriven and C. V. Sterling, "On cellular convection driven by surface-tension gradients-Effects of mean surface tension and surface viscosity," J. Fluid Mech. 19, 321 (1964).

${ }^{10}$ A. E. Hosoi and J. W. M. Bush, "Evaporative instabilities in climbing films," J. Fluid Mech. 442, 217 (2001).

${ }^{11}$ J. J. Xu and S. H. Davis, "Convective thermocapillary instabilities in liquid bridges," Phys. Fluids 27, 1102 (1984).

${ }^{12}$ H. Wang, S. V. Garimella, and J. Y. Murthy, "Characteristics of an evaporating thin film in a microchannel," Int. J. Heat Mass Transfer 50, 3933 (2007).

${ }^{13} \mathrm{~B}$. V. Derjaguin, "Dependence of the wetting tension on the meniscus curvature and the isotherm of the disjoining pressure of the liquid interlayer adjacent to it," J. Colloid Interface Sci. 71, 431 (1979).

${ }^{14}$ P. C. Wayner, Y. K. Kao, and L. V. Lacroix, "Interline heat-transfer coefficient of an evaporating wetting film," Int. J. Heat Mass Transfer 19, 487 (1976).

${ }^{15}$ P. C. Wayner, "Intermolecular forces in phase-change heat transfer: 1998 Kern award review," AIChE J. 45, 2055 (1999).

${ }^{16}$ H. B. Ma and G. P. Peterson, "Experimental investigation of the maximum heat transport in triangular grooves," J. Heat Transfer 118, 740 (1996).

${ }^{17}$ H. B. Ma and G. P. Peterson, "The minimum meniscus radius and capillary heat transport limit in micro heat pipes," J. Heat Transfer 120, 227 (1998).

${ }^{18}$ S. K. Wilson, S. H. Davis, and S. G. Bankoff, "The unsteady expansion and contraction of a long two-dimensional vapour bubble between superheated or subcooled parallel plates," J. Fluid Mech. 391, 1 (1999).

${ }^{19}$ A. Oron, S. G. Bankoff, and S. H. Davis, "Thermal singularities in film rupture," Phys. Fluids 8, 3433 (1996).

${ }^{20}$ W. M. Grissom and F. A. Wierum, "Liquid spray cooling of a heated surface," Int. J. Heat Mass Transfer 24, 261 (1981).

${ }^{21}$ A. G. Yiotis, A. G. Boudouvis, A. K. Stubos, I. N. Tsimpanogiannis, and Y. C. Yortsos, "Effect of liquid films on the isothermal drying of porous media," Phys. Rev. E 68, 037303 (2003).

${ }^{22}$ G. R. Schmidt, A. Nadarajah, T. J. Chung, and G. R. Karr, "Influence of 2-phase thermocapillary flow on liquid retention in microscopic pores," $\mathrm{J}$. Thermophys. Heat Transfer 9, 151 (1995).

${ }^{23}$ M. C. Limbourg-Fontaine, G. Petre, and J. C. Legros, "Texus 8 experiment: Effects of a surface tension minimum on thermocapillary convection," PCH, PhysicoChem. Hydrodyn. 6, 301 (1985).

${ }^{24}$ D. Villers and J. K. Platten, "Separation of Marangoni convection from gravitational convection in earth experiments," PCH, PhysicoChem. Hydrodyn. 8, 173 (1987).

${ }^{25}$ K. Wozniak, G. Wozniak, and T. Roesgen, "Particle-image-velocimetry applied to thermocapillary convection," Exp. Fluids 10, 12 (1990).

${ }^{26} \mathrm{G}$. Wozniak, K. Wozniak, and H. Bergelt, "On the influence of buoyancy on the surface tension driven flow around a bubble on a heated wall," Exp. Fluids 21, 181 (1996).

${ }^{27} \mathrm{~K}$. Wozniak, G. Wozniak, and T. Roesgen, "Experimental investigation of the thermocapillary flow around a bubble by means of laser-specklevelocimetry," Proc. SPIE 295, 337 (1989).

${ }^{28}$ A. D. Pline, M. P. Wernet, and K.-C. Hsieh, "Ground-based PIV and numerical flow visualization results from the surface-tension-driven convection experiment," Proc. SPIE 1557, 222 (1991)

${ }^{29}$ Y. Kamotani, S. Ostrach, and A. Pline, "Analysis of velocity data taken in surface-tension driven convection experiment in microgravity," Phys. Fluids 6, 3601 (1994).

${ }^{30}$ R. Savino and S. Fico, "Buoyancy and surface tension driven convection around a bubble," Phys. Fluids 18, 057104 (2006).

${ }^{31}$ C. A. Ward and F. Duan, "Turbulent transition of thermocapillary flow induced by water evaporation," Phys. Rev. E 69, 056308 (2004).

${ }^{32}$ O. E. Ruiz and W. Z. Black, "Evaporation of water droplets placed on a heated horizontal surface," J. Heat Transfer 124, 854 (2002).

${ }^{33}$ A. G. Kirdyashkin, "Thermogravitational and thermocapillary flows in a horizontal liquid layer under the conditions of a horizontal temperature gradient,” Int. J. Heat Mass Transfer 27, 1205 (1984).

${ }^{34}$ D. Villers and J. K. Platten, "Coupled buoyancy and Marangoni convection in acetone-experiments and comparison with numerical simulations," J. Fluid Mech. 234, 487 (1992).

${ }^{35}$ M. Behnia, F. Stella, and G. Guj, "A numerical study of 3-dimensional combined buoyancy and thermocapillary convection," Int. J. Multiphase Flow 21, 529 (1995).

${ }^{36}$ T. L. Bergman and S. Ramadhyani, "Combined buoyancy-driven and thermocapillary-driven convection in open square cavities," Numer. Heat Transfer 9, 441 (1986).

${ }^{37}$ C. Buffone, K. Sefiane, and J. R. E. Christy, "Experimental investigation of self-induced thermocapillary convection for an evaporating meniscus in capillary tubes using micro-particle image velocimetry," Phys. Fluids 17, 052104 (2005).

${ }^{38}$ J. G. Santiago, S. T. Wereley, C. D. Meinhart, D. J. Beebe, and R. J. Adrian, "A particle image velocimetry system for microfluidics," Exp. Fluids 25, 316 (1998).

${ }^{39}$ P. Chamarthy, H. K. Dhavaleswarapu, S. V. Garimella, J. Y. Murthy, and S. T. Wereley, "Visualization of convection patterns near an evaporating meniscus using $\mu \mathrm{PIV}$," in Proceedings of the 12th International Symposium on Flow Visualization, Göttingen, Germany, 10-14 September 2006, edited by I. Grant (Optimage, Edinburgh, 2006).

${ }^{40}$ L. Gui and S. T. Wereley, "A correlation-based continuous window-shift technique to reduce the peak-locking effect in digital PIV image evaluation," Exp. Fluids 32, 506 (2002).

${ }^{41}$ H. K. Dhavaleswarapu, P. Chamarthy, S. V. Garimella, J. Y. Murthy, and S. T. Wereley, "Experimental investigation of thermocapillary convection near an evaporating meniscus," in Proceedings of the ASME International Mechanical Engineering Congress and Exposition, Chicago, IL (ASME, New York, 2006), IMECE paper 2006-13901.

${ }^{42}$ J. Rice and A. Faghri, "A new computational method to track a liquid/ vapor interface with mass transfer, demonstrated on the concentration driven evaporation from a capillary tube and the Marangoni effect," in Proceedings of the ASME International Mechanical Engineering Congress and Exposition, Orlando, FL (ASME, New York, 2005), IMECE paper 2005-81433.

${ }^{43}$ H. Wang, S. V. Garimella, and J. Y. Murthy, "Transport from a volatile meniscus in a microtube," in Proceedings of the ASME International Me- 
chanical Engineering Congress and Exposition, Chicago, IL (ASME, New York, 2006), IMECE paper 2006-13898.

${ }^{44}$ C. N. Peiss, "Evaporation of small water drops maintained at constant volume," J. Appl. Phys. 65, 5235 (1989).

${ }^{45}$ K. Hisatake, S. Tanaka, and Y. Aizawa, "Evaporation rate of water in a vessel," J. Appl. Phys. 73, 7395 (1993).

${ }^{46} \mathrm{C}$. Buffone and K. Sefiane, "Investigation of thermocapillary convective patterns and their role in the enhancement of evaporation from pores," Int. J. Multiphase Flow 30, 1071 (2004).

${ }^{47}$ R. D. Deegan, O. Bakajin, T. F. Dupont, G. Huber, S. R. Nagel, and T. A. Witten, "Capillary flow as the cause of ring stains from dried liquid drops," Nature (London) 389, 827 (1997).

${ }^{48}$ R. D. Deegan, O. Bakajin, T. F. Dupont, G. Huber, S. R. Nagel, and T. A. Witten, "Contact line deposits in an evaporating drop," Phys. Rev. E 62, 756 (2000).

${ }^{49}$ G. Fang and C. A. Ward, "Temperature measured close to the interface of an evaporating liquid," Phys. Rev. E 59, 417 (1999).

${ }^{50} \mathrm{C}$. Buffone and K. Sefiane, "IR measurements of interfacial temperature during phase change in a confined environment," Exp. Therm. Fluid Sci. 29, 65 (2004)

${ }^{51}$ N. Kobayashi, "Steady convection caused by the temperature inhomogeneity in a cylindrical floating zone," Jpn. J. Appl. Phys., Part 1 27, 20 (1988).

${ }^{52}$ E. Koukan, G. Wozniak, K. Wozniak, and J. Siekmann, "Experimental study of flow fields around small gas bubbles under the combined action of buoyancy and thermocapillarity," Heat Mass Transfer 37, 437 (2001).

${ }^{53}$ R. J. Riley and G. P. Neitzel, "Instability of thermocapillary-buoyancy convection in shallow layers. Part 1. Characterization of steady and oscillatory instabilities," J. Fluid Mech. 359, 143 (1998).

${ }^{54}$ N. N. Lebedev and R. A. Silverman, Special Functions and Their Applications (Dover, New York, 1972). 
Physics of Fluids is copyrighted by the American Institute of Physics (AIP). Redistribution of journal material is subject to the AIP online journal license and/or AIP copyright. For more information, see http://ojps.aip.org/phf/phfor.jsp 